Supplemental materials for:

\title{
The Direct Acyl-Alkylation of Arynes
}

Uttam K. Tambar and Brian M. Stoltz*

The Arnold and Mabel Beckman Laboratories of Chemical Synthesis, Division of Chemistry and Chemical Engineering, California Institute of Technology, Pasadena, California 91125, USA

\section{Table of Contents:}

Materials and Methods

Synthesis of Aryne Precursors

Synthesis of $\beta$-Ketoester Substrates

Representative Procedure for the Acyl-Alkylation of Arynes

Spectral Data

Independent Chemical Correlation / Structural Proof

References
1

2

3

3

4

11

12

Materials and Methods. Unless stated otherwise, reactions were performed in flame-dried glassware sealed with rubber septa under a nitrogen atmosphere using dry, deoxygenated solvents. Commercially obtained reagents were used as received. Cesium fluoride was purchased from the Sigma-Aldrich Chemical Company, Milwaukee, WI. Solvents were dried by passage through an activated alumina column under argon. Liquids and solutions were transferred via syringe. Reaction temperatures were controlled by an IKAmag temperature modulator. Thin-layer chromatography (TLC) was performed using E. Merck silica gel 60 F254 precoated plates $(0.25 \mathrm{~mm})$ and visualized using a combination of UV, anisaldehyde, ceric ammonium molybdate, and potassium permanganate staining. ICN silica gel (particle size $0.032-0.063 \mathrm{~mm}$ ) was used for flash chromatography. ${ }^{1} \mathrm{H}$ NMR spectra were recorded on a Varian Mercury 300 (at $300 \mathrm{MHz}$ ) or a Varian Inova 500 (at $500 \mathrm{MHz}$ ) and are reported relative to $\mathrm{Me}_{4} \mathrm{Si}(\delta 0.0)$. Data for ${ }^{1} \mathrm{H}$ NMR spectra are reported as follows: chemical shift $(\delta \mathrm{ppm})$, multiplicity, coupling constant $(\mathrm{Hz})$ and integration. ${ }^{13} \mathrm{C}$ NMR spectra were recorded on a Varian Mercury 300 (at $75 \mathrm{MHz}$ ), or a Varian Inova 500 (at $125 \mathrm{MHz}$ ) and are reported relative to $\mathrm{Me}_{4} \mathrm{Si}(\delta$ 0.0). Data for ${ }^{13} \mathrm{C}$ NMR spectra are reported in terms of chemical shift. ${ }^{19} \mathrm{~F}$ NMR spectra were recorded on a 
Varian Mercury 300 (at $300 \mathrm{MHz}$ ) and are reported relative to $\mathrm{CF}_{3} \mathrm{COOH}(\delta-76.54)$. Data for ${ }^{19} \mathrm{~F} \mathrm{NMR}$ spectra are reported in terms of chemical shift. IR spectra were recorded on a Perkin Elmer Spectrum BXII spectrometer and are reported in terms of frequency of absorption $\left(\mathrm{cm}^{-1}\right)$. High resolution mass spectra were obtained from the California Institute of Technology Mass Spectral Facility.

\section{Synthesis of Aryne Precursors}

Aryne precursor 2-(trimethylsilyl)phenyl trifluoromethanesulfonate (1) was purchased from the Sigma-Aldrich Chemical Company, Milwaukee, WI. Aryne precursors 3-methoxy-2-(trimethylsilyl)phenyl trifluoromethanesulfonate (10a) ${ }^{1}$ and 4-methyl-2-(trimethylsilyl)phenyl trifluoromethanesulfonate $(\mathbf{1 0 b})^{2}$ were prepared according to literature procedures.
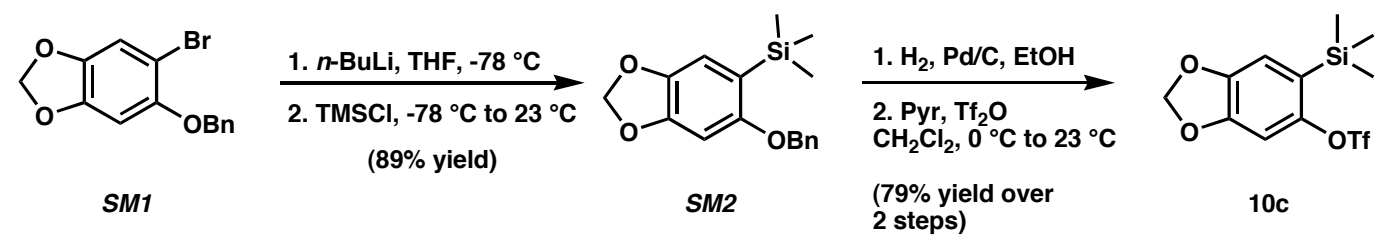

SM2. A flame-dried reaction flask equipped with a magnetic stir bar was charged with benzyl protected sesamol $\boldsymbol{S M I}^{3}$ (325 mg, $1.06 \mathrm{mmol}, 1.0$ equiv) in THF $(3.5 \mathrm{~mL}, 0.3 \mathrm{M})$, and the mixture was cooled to $-78{ }^{\circ} \mathrm{C}$ in a dry ice/acetone bath. A $2.5 \mathrm{M}$ solution of $n$-BuLi in hexanes $(634 \mu \mathrm{L}, 1.59 \mathrm{mmol}, 1.5$ equiv) was added slowly at $-78^{\circ} \mathrm{C}$. After 15 minutes, TMSCl $(200 \mu \mathrm{L}, 1.59 \mathrm{mmol}, 1.5$ equiv $)$ was added slowly at $-78^{\circ} \mathrm{C}$. After 5 minutes, the cold bath was removed and the reaction mixture was allowed to warm to $23{ }^{\circ} \mathrm{C}$. After 15 minutes, the mixture was quenched with saturated aqueous $\mathrm{NH}_{4} \mathrm{Cl}(5 \mathrm{~mL})$. The aqueous layer was extracted with $\mathrm{Et}_{2} \mathrm{O}(3 \times 5$ $\mathrm{mL}$ ). The organics were combined and dried over $\mathrm{Na}_{2} \mathrm{SO}_{4}$. After filtration, the residue was concentrated under reduced pressure. Purification by flash chromatography (10:1 hexanes/EtOAc eluent) provided aryl intermediate SM2 (282 mg, 89\% yield) as a clear oil: $\mathrm{R}_{\mathrm{F}} 0.57$ (3:1 hexanes/EtOAc); ${ }^{1} \mathrm{H}$ NMR (300 MHz, $\mathrm{CDCl}_{3}$ ) $\delta$ 7.45-7.29 $(\mathrm{m}, 5 \mathrm{H}), 6.85(\mathrm{~s}, 1 \mathrm{H}), 6.53(\mathrm{~s}, 1 \mathrm{H}), 5.91(\mathrm{~s}, 2 \mathrm{H}), 5.01(\mathrm{~s}, 2 \mathrm{H}), 0.23(\mathrm{~s}, 9 \mathrm{H}) ;{ }^{13} \mathrm{C} \mathrm{NMR}\left(75 \mathrm{MHz}, \mathrm{CDCl}_{3}\right) \delta$ 159.2, 149.6, 141.5, 137.3, 128.7, 128.0, 127.5, 119.3, 113.7, 101.3, 95.2, 71.0, -0.5; IR (film) 2953, 2894, 1606, 1502, 1473, 1410, 1386, 1243, 1177, $1042 \mathrm{~cm}^{-1}$; HRMS (EI ${ }^{+}$) calc'd for $\left[\mathrm{C}_{17} \mathrm{H}_{20} \mathrm{O}_{3} \mathrm{Si}^{+}\right]^{+}: \mathrm{m} / z \quad 300.1182$, found 300.1187 .

Aryne Precursor 10c. A reaction flask equipped with a magnetic stir bar was charged with aryl intermediate SM2 (1.24 g, $4.13 \mathrm{mmol}, 1$ equiv) in absolute EtOH $(17 \mathrm{~mL}, 0.25 \mathrm{M})$. To this mixture was added $10 \% \mathrm{Pd} / \mathrm{C}$ (440 mg, $0.413 \mathrm{mmol}, 0.1$ equiv), and the reaction vessel was stirred at room temperature under a balloon of $\mathrm{H}_{2}$ (1 atm). After 14 hours the mixture was filtered through a short plug of celite $\left(\mathrm{Et}_{2} \mathrm{O}\right.$ eluent), and the solvent was 
evaporated under reduced pressure to afford a colorless oil, which was used immediately without further purification.

A flame-dried reaction flask equipped with a magnetic stir bar was charged with the ortho-silyl phenol in $\mathrm{CH}_{2} \mathrm{Cl}_{2}$ (20 mL, 0.2 equiv), and the mixture was cooled to $0{ }^{\circ} \mathrm{C}$. Pyridine $\left(1.05 \mathrm{~mL}, 13 \mathrm{mmol}, 3\right.$ equiv) and $\mathrm{Tf}_{2} \mathrm{O}$ $(1.46 \mathrm{~mL}, 8.67 \mathrm{mmol}, 2$ equiv) were sequentially added, and the reaction mixture was allowed to warm to room temperature. After 5.5 hours the mixture was extracted with $\mathrm{H}_{2} \mathrm{O}(20 \mathrm{~mL})$. The aqueous layer was backextracted with $\mathrm{CH}_{2} \mathrm{Cl}_{2}(3 \times 10 \mathrm{~mL})$. The organics were combined and dried over $\mathrm{Na}_{2} \mathrm{SO}_{4}$. After filtration, the residue was concentrated under reduced pressure. Purification by flash chromatography (10:1 hexanes/ $\mathrm{CH}_{2} \mathrm{Cl}_{2}$ eluent) provided aryne precursor 10c $\left(1.116 \mathrm{~g}, 79 \%\right.$ yield) as a clear oil: $\mathrm{R}_{\mathrm{F}} 0.54$ (3:1 hexanes/EtOAc); ${ }^{1} \mathrm{H}$ NMR $\left(500 \mathrm{MHz}, \mathrm{CDCl}_{3}\right)_{-} 6.88(\mathrm{~s}, 1 \mathrm{H}), 6.84(\mathrm{~s}, 1 \mathrm{H}), 6.03(\mathrm{~s}, 2 \mathrm{H}), 0.33(\mathrm{~s}, 9 \mathrm{H}) ;{ }^{13} \mathrm{C} \mathrm{NMR}\left(125 \mathrm{MHz}, \mathrm{CDCl}_{3}\right)_{-}$ 149.7, 148.8, 147.1, 125.1, 113.4, 113.4, 102.6, 102.5, $-0.5 ;{ }^{19} \mathrm{~F}$ NMR (300 MHz, $\left.\mathrm{CDCl}_{3}\right)_{-}$-74.63; IR (film)

2960, 2903, 1479, 1422, 1247, 1216, 1141, 984, $843 \mathrm{~cm}^{-1}$; HRMS $\left(\mathrm{EI}^{+}\right)$calc'd for $\left[\mathrm{C}_{11} \mathrm{H}_{13} \mathrm{O}_{5} \mathrm{~F}_{3} \mathrm{SiS}^{+}: \mathrm{m} / z\right.$ 342.0205 , found 342.0211 .

\section{Synthesis of $\beta$-Ketoester Substrates}

Methyl acetoacetate (8a), ethyl 2-methyl-3-oxobutanoate (2), ethyl 2-oxocyclopentanecarboxylate (12b), and methyl 2-oxocycloheptanecarboxylate (12f) were purchased from the Sigma-Aldrich Chemical Company, Milwaukee, WI. Substrates $8 \mathrm{~b},{ }^{4} \mathbf{8 c},{ }^{5} 8 \mathrm{~d},{ }^{6} \mathbf{8 e},{ }^{7} \mathbf{8 f},{ }^{8} \mathbf{8 g},{ }^{9} \mathbf{8 h},{ }^{10} \mathbf{1 2 b},{ }^{11} \mathbf{1 2 c},{ }^{12}$ and $\mathbf{1 2 d}{ }^{13}$ were prepared according to literature procedures.

Representative procedure for the acyl-alkylation of arynes (Tables 1, 2, and 3): A flame-dried long reaction tube equipped with a magnetic stir bar was charged with acetonitrile $(2 \mathrm{~mL})$. Methyl acetoacetate (8a) (43.2 $\mu \mathrm{L}, 0.4 \mathrm{mmol}, 1.0$ equiv), 2-(trimethylsilyl)phenyl trifluoromethanesulfonate (1) (121.4 $\mu \mathrm{L}, 0.5 \mathrm{mmol}$, 1.25 equiv), and cesium fluoride ( $152 \mathrm{mg}, 1.0 \mathrm{mmol}, 2.5$ equiv) were sequentially added to the flask. A septum was placed on the reaction vessel, and the mixture was then heated at $80{ }^{\circ} \mathrm{C}$ for $45-60$ minutes. When benzyne precursor 1 was consumed by TLC analysis, the mixture was extracted with brine $(4 \mathrm{~mL})$. The aqueous layer was back-extracted with $\mathrm{Et}_{2} \mathrm{O}(3 \times 4 \mathrm{~mL})$. The organics were combined and dried over $\mathrm{Na}_{2} \mathrm{SO}_{4}$. After filtration, the residue was concentrated under reduced pressure and purified by flash chromatography. 


\section{Spectral Data}

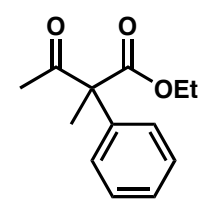

6. Purification by flash chromatography (20:1 hexanes/EtOAc eluent) provided the desired acyl-alkylation product (37.1 mg, $42 \%$ yield) as a clear oil: $\mathrm{R}_{\mathrm{F}} 0.36$ (3:1 hexanes/EtOAc); ${ }^{1} \mathrm{H}$ NMR $\left(300 \mathrm{MHz}, \mathrm{CDCl}_{3}\right)$ _ $7.40-$ 7.27 (m, 5H), 4.26 (q, $J=7.1 \mathrm{~Hz}, 2 \mathrm{H}), 2.10$ (s, 3H), 1.77 (s, 3H), 1.28 (t, $J=7.2 \mathrm{~Hz}, 3 \mathrm{H}) ;{ }^{13} \mathrm{C}$ NMR $(75 \mathrm{MHz}$, $\mathrm{CDCl}_{3}$ )_205.1, 172.1, 138.8, 128.8, 127.9, 127.5, 64.9, 61.8, 27.4, 21.6, 14.2; IR (film) 2986, 1715, $1251 \mathrm{~cm}^{-1}$; HRMS $\left(\mathrm{EI}^{+}\right)$calc'd for $\left[\mathrm{C}_{13} \mathrm{H}_{16} \mathrm{O}_{3}\right]^{+}: \mathrm{m} / \mathrm{z} 220.1100$, found 220.1089 .

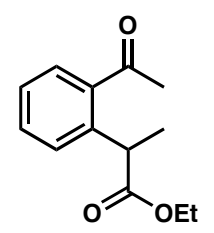

7. Purification by flash chromatography (20:1 hexanes/EtOAc eluent) provided the desired acyl-alkylation product (46.4 mg, 53\% yield) as a clear oil: $\mathrm{R}_{\mathrm{F}} 0.42$ (3:1 hexanes/EtOAc); ${ }^{1} \mathrm{H}$ NMR $\left(300 \mathrm{MHz}, \mathrm{CDCl}_{3}\right)$ _ 7.68 (dd, $J=7.8,1.5 \mathrm{~Hz}, 1 \mathrm{H}), 7.49-7.29(\mathrm{~m}, 3 \mathrm{H}), 4.41$ (q, $J=7.0 \mathrm{~Hz}, 1 \mathrm{H}), 4.17-4.05(\mathrm{~m}, 2 \mathrm{H}), 2.59(\mathrm{~s}, 3 \mathrm{H}), 1.50(\mathrm{~d}$, $J=7.2 \mathrm{~Hz}, 3 \mathrm{H}), 1.19(\mathrm{t}, J=7.0 \mathrm{~Hz}, 3 \mathrm{H}) ;{ }^{13} \mathrm{C} \mathrm{NMR}\left(75 \mathrm{MHz}, \mathrm{CDCl}_{3}\right) \_202.4,174.8,140.4,137.8,132.0$, 129.3, 128.8, 126.9, 60.8, 41.7, 29.9, 18.4, 14.3; IR (film) 2982, 1731, 1687, $1201 \mathrm{~cm}^{-1}$; HRMS (EI ${ }^{+}$) calc'd for $\left[\mathrm{C}_{13} \mathrm{H}_{16} \mathrm{O}_{3}\right]^{+}: m / z 220.1100$, found 220.1091 .

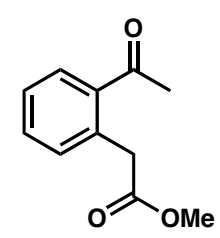

9a. Purification by flash chromatography (10:1 hexanes/EtOAc eluent) provided the desired acyl-alkylation product (69 mg, 90\% yield) as a clear oil: $\mathrm{R}_{\mathrm{F}} 0.46$ (3:1 hexanes/EtOAc); ${ }^{1} \mathrm{H}$ NMR (300 MHz, $\left.\mathrm{CDCl}_{3}\right) \delta 7.79$ $(\mathrm{dd}, J=7.6,1.5 \mathrm{~Hz}, 1 \mathrm{H}), 7.47-7.33(\mathrm{~m}, 2 \mathrm{H}), 7.24-7.20(\mathrm{~m}, 1 \mathrm{H}), 3.91(\mathrm{~s}, 2 \mathrm{H}), 3.66(\mathrm{~s}, 3 \mathrm{H}), 2.56(\mathrm{~s}, 3 \mathrm{H}) ;{ }^{13} \mathrm{C}$ NMR (75 MHz, $\left.\mathrm{CDCl}_{3}\right)_{-}$201.3, 172.0, 137.1, 134.4, 132.8, 132.1, 130.1, 127.5, 51.9, 40.2, 28.8; IR (film) 3001, 2952, 1739, $1683 \mathrm{~cm}^{-1}$; HRMS $\left(\mathrm{EI}^{+}\right)$calc'd for $\left[\mathrm{C}_{11} \mathrm{H}_{12} \mathrm{O}_{3}\right]^{+}: \mathrm{m} / z$ 192.0787, found 192.0787. 


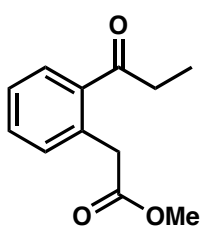

9b. Purification by flash chromatography (15:1 hexanes/EtOAc eluent) provided the desired acyl-alkylation product (64.7 mg, 78\% yield) as a clear oil: $\mathrm{R}_{\mathrm{F}} 0.32$ (3:1 hexanes/EtOAc); ${ }^{1} \mathrm{H}$ NMR (300 MHz, $\left.\mathrm{CDCl}_{3}\right){ }_{-} 7.77$ $(\mathrm{dd}, J=7.6,1.5 \mathrm{~Hz}, 1 \mathrm{H}), 7.48-7.31(\mathrm{~m}, 2 \mathrm{H}), 7.26-7.22(\mathrm{~m}, 1 \mathrm{H}), 3.92(\mathrm{~s}, 2 \mathrm{H}), 3.68(\mathrm{~s}, 3 \mathrm{H}), 2.97(\mathrm{q}, J=7.3 \mathrm{~Hz}$, 2H), $1.18(\mathrm{t}, J=7.3 \mathrm{~Hz}, 3 \mathrm{H}) ;{ }^{13} \mathrm{C}$ NMR $\left(75 \mathrm{MHz}, \mathrm{CDCl}_{3}\right)$ _ 204.4, 172.2, 137.7, 134.2, 132.7, 131.8, 129.1, 127.5, 52.0, 40.1, 34.0, 8.4; IR (film) 2979, 2951, 1739, 1685, 1225, $1165 \mathrm{~cm}^{-1}$; HRMS (EI ${ }^{+}$) calc'd for $\left[\mathrm{C}_{12} \mathrm{H}_{14} \mathrm{O}_{3}\right]^{+}: m / z 206.0943$, found 206.0933.

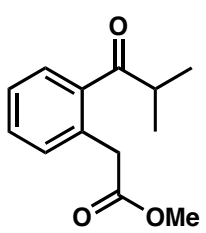

9c. Purification by flash chromatography (15:1 hexanes/EtOAc eluent) provided the desired acyl-alkylation product (71.9 mg, 84\% yield) as a clear oil: $\mathrm{R}_{\mathrm{F}} 0.35$ (3:1 hexanes/EtOAc); ${ }^{1} \mathrm{H}$ NMR (300 $\left.\mathrm{MHz}, \mathrm{CDCl}_{3}\right){ }_{-} 7.78$ $(\mathrm{d}, J=7.4 \mathrm{~Hz}, 1 \mathrm{H}), 7.53-7.25(\mathrm{~m}, 3 \mathrm{H}), 3.91(\mathrm{~s}, 2 \mathrm{H}), 3.72(\mathrm{~s}, 3 \mathrm{H}), 3.56-3.44(\mathrm{~m}, 1 \mathrm{H}), 1.21(\mathrm{~d}, J=6.6 \mathrm{~Hz}, 6 \mathrm{H})$; ${ }^{13} \mathrm{C}$ NMR $\left(75 \mathrm{MHz}, \mathrm{CDCl}_{3}\right)_{2}$ 207.9, 172.2, 137.2, 134.7, 132.9, 131.7, 128.9, 127.5, 52.0, 40.0, 37.7, 19.0; IR (film) 2973, 1741, 1683, 1229, $1165 \mathrm{~cm}^{-1}$; HRMS $\left(\mathrm{EI}^{+}\right)$calc'd for $\left[\mathrm{C}_{13} \mathrm{H}_{16} \mathrm{O}_{3}\right]^{+}: \mathrm{m} / z$ 220.1100, found 220.1094.

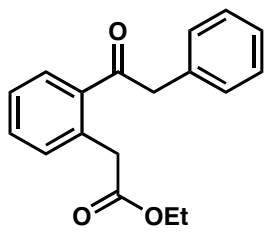

9d. Purification by flash chromatography (20:1 hexanes/EtOAc eluent) provided the desired acyl-alkylation product (95.5 mg, 85\% yield) as a clear oil: $\mathrm{R}_{\mathrm{F}} 0.35$ (3:1 hexanes/EtOAc); ${ }^{1} \mathrm{H}$ NMR $\left(300 \mathrm{MHz}, \mathrm{CDCl}_{3}\right){ }_{-} 7.86$ (dd, $J=7.6,1.5 \mathrm{~Hz}, 1 \mathrm{H}), 7.49-7.24(\mathrm{~m}, 8 \mathrm{H}), 4.28(\mathrm{~s}, 2 \mathrm{H}), 4.14(\mathrm{q}, J=7.2 \mathrm{~Hz}, 2 \mathrm{H}), 3.92(\mathrm{~s}, 2 \mathrm{H}), 1.25(\mathrm{t}, J=7.0$ $\mathrm{Hz}, 3 \mathrm{H}) ;{ }^{13} \mathrm{C} \mathrm{NMR}\left(75 \mathrm{MHz}, \mathrm{CDCl}_{3}\right)$ _ 201.1, 171.7, 137.6, 134.7, 132.8, 131.9, 129.8, 129.4, 128.8, 128.7, 127.5, 127.0, 60.9, 47.8, 40.1, 14.4; IR (film) 3362, 2982, 1732, 1690, 1216, $1175 \mathrm{~cm}^{-1}$; HRMS (EI ${ }^{+}$) calc'd for $\left[\mathrm{C}_{18} \mathrm{H}_{18} \mathrm{O}_{3}\right]^{+}: \mathrm{m} / z$ 282.1256, found 282.1266. 


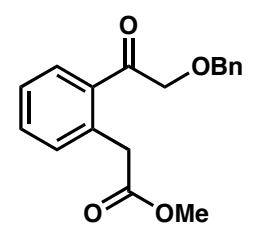

9e. Purification by flash chromatography (15:1 hexanes/EtOAc eluent) provided the desired acyl-alkylation product (66.2 mg, 53\% yield) as a clear oil: $\mathrm{R}_{\mathrm{F}} 0.28$ (3:1 hexanes/EtOAc); ${ }^{1} \mathrm{H}$ NMR $\left(300 \mathrm{MHz}, \mathrm{CDCl}_{3}\right){ }_{-} 7.66$ (dd, $J=7.7,1.3 \mathrm{~Hz}, 1 \mathrm{H}), 7.48$ (app. dt, $J=7.6,1.4 \mathrm{~Hz}, 1 \mathrm{H}), 7.41-7.27$ (m, 7H), 4.68 (s, 2H), 4.67 (s, $2 \mathrm{H}), 3.97$ (s, 2H), 3.69 (s, 3H); ${ }^{13} \mathrm{C}$ NMR (75 MHz, $\left.\mathrm{CDCl}_{3}\right)_{\text {_ }}$ 200.1, 172.1, 137.5, 135.3, 134.7, 132.9, 132.4, 129.0, 128.7, 128.3, 128.2, 127.5, 73.6, 73.4, 52.2, 39.7; IR (film) 3030, 2950, 1736, 1700, 1230, 1213, $1167,1110 \mathrm{~cm}^{-}$ '; HRMS $\left(\mathrm{EI}^{+}\right)$calc'd for $(\mathrm{M}-\mathrm{H})\left[\mathrm{C}_{18} \mathrm{H}_{17} \mathrm{O}_{4}\right]^{+}: \mathrm{m} / z$ 297.1127, found 297.1136.

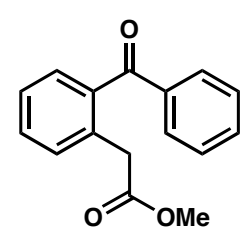

9f. Purification by flash chromatography (10:1 hexanes/EtOAc eluent) provided the desired acyl-alkylation product (101 mg, 99\% yield) as a clear oil: $\mathrm{R}_{\mathrm{F}} 0.38$ (3:1 hexanes/EtOAc); ${ }^{1} \mathrm{H}$ NMR $\left(300 \mathrm{MHz}, \mathrm{CDCl}_{3}\right)$ _ $7.84-$ $7.79(\mathrm{~m}, 1 \mathrm{H}), 7.61-7.54(\mathrm{~m}, 1 \mathrm{H}), 7.50-7.30(\mathrm{~m}, 7 \mathrm{H}), 3.90(\mathrm{~s}, 2 \mathrm{H}), 3.55(\mathrm{~s}, 3 \mathrm{H}) ;{ }^{13} \mathrm{C} \mathrm{NMR}\left(75 \mathrm{MHz} \mathrm{CDCl}_{3}\right)_{-}$ 198.2, 171.8, 138.4, 137.9, 134.1, 133.1, 131.9, 131.0, 130.5, 130.2, 128.4, 126.7, 52.0, 38.8; IR (film) 2951, $1739,1662,1270 \mathrm{~cm}^{-1}$; HRMS $\left(\mathrm{EI}^{+}\right)$calc'd for $\left[\mathrm{C}_{16} \mathrm{H}_{14} \mathrm{O}_{3}\right]^{+}: \mathrm{m} / z$ 254.0943, found 254.0952 .

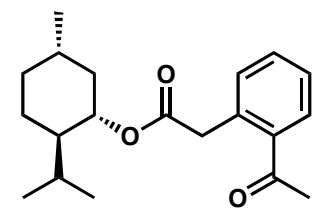

9g. Purification by flash chromatography (15:1 hexanes/EtOAc eluent) provided the desired acyl-alkylation product (90.7 mg, 72\% yield) as a clear oil: $\mathrm{R}_{\mathrm{F}} 0.50$ (3:1 hexanes/EtOAc); ${ }^{1} \mathrm{H}$ NMR (300 MHz, $\left.\mathrm{CDCl}_{3}\right){ }_{-} 7.81$ $(\mathrm{dd}, J=7.4,1.6 \mathrm{~Hz}, 1 \mathrm{H}), 7.51-7.34(\mathrm{~m}, 2 \mathrm{H}), 7.28(\mathrm{dd}, J=7.4,1.1 \mathrm{~Hz}, 1 \mathrm{H}), 4.76-4.66(\mathrm{~m}, 1 \mathrm{H}), 3.97$ (s, $2 \mathrm{H})$, $2.62(\mathrm{~s}, 3 \mathrm{H}), 2.11-2.02(\mathrm{~m}, 1 \mathrm{H}), 1.98-1.86(\mathrm{~m}, 1 \mathrm{H}), 1.75-1.64(\mathrm{~m}, 2 \mathrm{H}), 1.58-1.33(\mathrm{~m}, 3 \mathrm{H}), 1.11-1.00(\mathrm{~m}, 2 \mathrm{H})$, $0.93(\mathrm{~d}, J=4.3 \mathrm{~Hz}, 3 \mathrm{H}), 0.91(\mathrm{~d}, J=5.1 \mathrm{~Hz}, 3 \mathrm{H}), 0.77(\mathrm{~d}, J=6.9 \mathrm{~Hz}, 3 \mathrm{H}) ;{ }^{13} \mathrm{C} \mathrm{NMR}\left(75 \mathrm{MHz} \mathrm{CDCl}_{3}\right)$ 201.4, 171.3, 137.7, 134.6, 132.7, 131.9, 129.8, 127.4, 74.8, 47.2, 41.0, 40.6, 34.4, 31.6, 29.0, 26.3, 23.6, 22.2, 20.9, 16.5; IR (film) 2955, 2929, 2870, 1732 1687, 1258, $1172 \mathrm{~cm}^{-1}$; HRMS (EI ${ }^{+}$) calc'd for $\left[\mathrm{C}_{20} \mathrm{H}_{28} \mathrm{O}_{3}\right]^{+}: \mathrm{m} / z$ 316.2039 , found 316.2034 . 


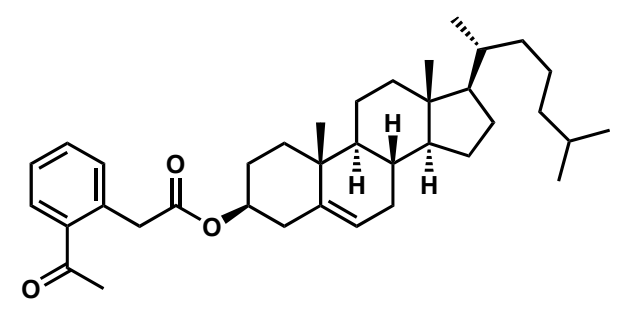

9h. Purification by flash chromatography (15:1 hexanes/EtOAc eluent) provided the desired acyl-alkylation product $\left(163.5 \mathrm{mg}, 75 \%\right.$ yield) as a clear oil: $\mathrm{R}_{\mathrm{F}} 0.50$ (3:1 hexanes/EtOAc); ${ }^{1} \mathrm{H}$ NMR $\left(300 \mathrm{MHz}, \mathrm{CDCl}_{3}\right) \ldots$ $(\mathrm{dd}, J=7.6,1.5 \mathrm{~Hz}, 1 \mathrm{H}), 7.48-7.33(\mathrm{~m}, 2 \mathrm{H}), 7.24(\mathrm{dd}, J=7.6,1.0 \mathrm{~Hz}, 1 \mathrm{H}), 5.36(\mathrm{~d}, J=5.1 \mathrm{~Hz}, 1 \mathrm{H}), 4.72-4.55$ $(\mathrm{m}, 1 \mathrm{H}), 3.91(\mathrm{~d}, J=1.6 \mathrm{~Hz}, 2 \mathrm{H}), 2.59(\mathrm{~s}, 3 \mathrm{H}), 2.35(\mathrm{~d}, J=7.7 \mathrm{~Hz}, 2 \mathrm{H}), 2.05-1.76(\mathrm{~m}, 5 \mathrm{H}), 1.70-1.05(\mathrm{~m}$, 21H), $1.02(\mathrm{~s}, 3 \mathrm{H}), 0.91(\mathrm{~d}, J=6.4 \mathrm{~Hz}, 3 \mathrm{H}), 0.86(\mathrm{dd}, J=6.5,1.2 \mathrm{~Hz}, 6 \mathrm{H}), 0.67(\mathrm{~s}, 3 \mathrm{H}) ;{ }^{13} \mathrm{C}$ NMR $(75 \mathrm{MHz}$, $\left.\mathrm{CDCl}_{3}\right)_{2} 201.3,171.2,139.9,137.5,134.7,132.8,132.1,130.0,127.5,122.7,74.6,56.9,56.3,50.2,42.5,40.7$, 39.9, 39.7, 38.2, 37.2, 36.8, 36.4, 36.0, 32.1, 32.0, 29.0, 28.4, 28.2, 27.9, 24.5, 24.0, 23.0, 22.8, 21.2, 19.5, 18.9, 12.0; IR (film) 3451, 2946, 2868, 1732, 1686, 1258, $1170 \mathrm{~cm}^{-1}$; HRMS (EI ${ }^{+}$) calc'd for $\left[\mathrm{C}_{37} \mathrm{H}_{54} \mathrm{O}_{3}\right]^{+}: \mathrm{m} / \mathrm{z}$ 546.4073 , found 546.4080 .

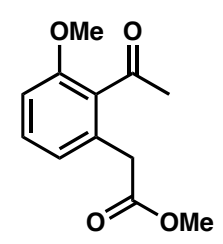

11a. Purification by flash chromatography (15:1 hexanes/EtOAc eluent) provided the desired acyl-alkylation product ( $84.6 \mathrm{mg}, 95 \%$ yield) as a clear oil: $\mathrm{R}_{\mathrm{F}} 0.29$ (3:1 hexanes/EtOAc); ${ }^{1} \mathrm{H}$ NMR $\left(300 \mathrm{MHz}, \mathrm{CDCl}_{3}\right)_{-} 7.30$ $(\mathrm{d}, J=8.2 \mathrm{~Hz}, 1 \mathrm{H}), 6.90-6.81(\mathrm{~m}, 2 \mathrm{H}), 3.84(\mathrm{~s}, 3 \mathrm{H}), 3.68(\mathrm{~s}, 2 \mathrm{H}), 3.66(\mathrm{~s}, 3 \mathrm{H}), 2.53(\mathrm{~s}, 3 \mathrm{H}) ;{ }^{13} \mathrm{C}$ NMR $(75$ $\mathrm{MHz}, \mathrm{CDCl}_{3}{ }_{2}$ 205.3, 171.9, 157.3, 132.7, 131.2, 130.8, 123.6, 110.4, 55.8, 52.2, 38.3, 32.3; IR (film) 3005, 2952, 2842, 1738, 1691, 1598, 1583, 1471, 1438, 1351, $1267 \mathrm{~cm}^{-1}$; HRMS $\left(\mathrm{EI}^{+}\right)$calc'd for $\left[\mathrm{C}_{12} \mathrm{H}_{14} \mathrm{O}_{4}\right]^{+}: \mathrm{m} / z$ 222.0892 , found 222.0892 .

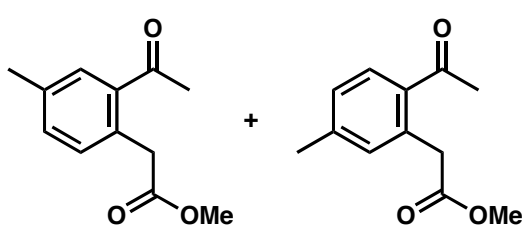

11b. Purification by flash chromatography (15:1 hexanes/EtOAc eluent) provided the desired acyl-alkylation products as a mixture of inseparable isomers $\left(67.9 \mathrm{mg}, 82 \%\right.$ yield) as a clear oil: $\mathrm{R}_{\mathrm{F}} 0.26$ (3:1 hexanes/EtOAc); ${ }^{1} \mathrm{H}$ NMR $\left(300 \mathrm{MHz}, \mathrm{CDCl}_{3}\right)_{-} 7.74(\mathrm{~d}, J=8.0 \mathrm{~Hz}, 1 \mathrm{H}), 7.61(\mathrm{~s}, 1 \mathrm{H}), 7.27(\mathrm{~d}, J=7.5 \mathrm{~Hz}, 1 \mathrm{H}), 7.19(\mathrm{~d}, J=7.9$ $\mathrm{Hz}, 1 \mathrm{H}), 7.13$ (d, $J=7.7 \mathrm{~Hz}, 1 \mathrm{H}), 7.05$ (s, 1H), 3.90 (s, 2H), 3.89 (s, 2H), 3.69 (s, 3H), 3.68 (s, 3H), 2.58 (s, $3 \mathrm{H}), 2.56(\mathrm{~s}, 3 \mathrm{H}), 2.40(\mathrm{~s}, 3 \mathrm{H}), 2.38(\mathrm{~s}, 3 \mathrm{H}) ;{ }^{13} \mathrm{C} \mathrm{NMR}\left(75 \mathrm{MHz}, \mathrm{CDCl}_{3}\right)_{-}$201.5, 200.7, 172.4, 172.3, 143.0, 
137.3, 137.1, 134.9, 134.3, 133.9, 132.9, 132.7, 131.5, 130.9, 130.8, 129.6, 128.2, 52.0, 40.6, 39.9, 28.9, 28.8, 21.6, 21.2; IR (film) 2952, 1740, $1680 \mathrm{~cm}^{-1}$; HRMS $\left(\mathrm{EI}^{+}\right.$) calc'd for $\left[\mathrm{C}_{12} \mathrm{H}_{14} \mathrm{O}_{3}\right]^{+}: \mathrm{m} / z$ 206.0943, found 206.0945 .

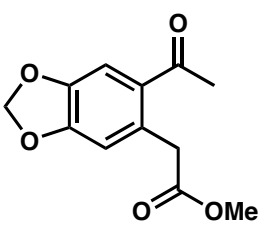

11c. Purification by flash chromatography ( $7: 1$ hexanes/EtOAc eluent) provided the desired acyl-alkylation product $\left(70.7 \mathrm{mg}, 75 \%\right.$ yield) as a clear oil: $\mathrm{R}_{\mathrm{F}} 0.18$ (3:1 hexanes/EtOAc); ${ }^{1} \mathrm{H}$ NMR $\left(300 \mathrm{MHz}, \mathrm{CDCl}_{3}\right)$ _ 7.29 $(\mathrm{s}, 1 \mathrm{H}), 6.70(\mathrm{~s}, 1 \mathrm{H}), 6.03(\mathrm{~s}, 2 \mathrm{H}), 3.84(\mathrm{~s}, 2 \mathrm{H}), 3.69(\mathrm{~s}, 3 \mathrm{H}), 2.51(\mathrm{~s}, 3 \mathrm{H}) ;{ }^{13} \mathrm{C}$ NMR $\left(75 \mathrm{MHz}, \mathrm{CDCl}_{3}\right)_{-}$ 199.0, 172.2, 150.6, 146.9, 131.2, 130.7, 113.0, 110.5, 102.2, 52.0, 40.7, 28.9; IR (film) 1738, 1678, 1613, 1507, $1491,1375,1274,1245 \mathrm{~cm}^{-1}$; HRMS $\left(\mathrm{EI}^{+}\right)$calc'd for $\left[\mathrm{C}_{12} \mathrm{H}_{12} \mathrm{O}_{5}\right]^{+}: \mathrm{m} / z 236.0685$, found 236.0692 .

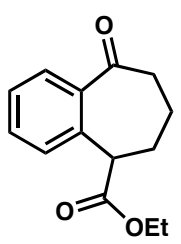

13a. Purification by flash chromatography (15:1 hexanes/EtOAc eluent) provided the desired acyl-alkylation product (46.9 mg, 51\% yield) as a clear oil: $\mathrm{R}_{\mathrm{F}} 0.32$ (3:1 hexanes/EtOAc); ${ }^{1} \mathrm{H}$ NMR $\left(300 \mathrm{MHz}, \mathrm{CDCl}_{3}\right)$ _ 7.67 (dd, $J=7.4,1.6 \mathrm{~Hz}, 1 \mathrm{H}), 7.51-7.40(\mathrm{~m}, 1 \mathrm{H}), 7.42-7.35(\mathrm{~m}, 1 \mathrm{H}), 7.22-7.18(\mathrm{~m}, 1 \mathrm{H}), 4.25-4.16(\mathrm{~m}, 2 \mathrm{H}), 4.02$ (dd, $J=7.4,5.6 \mathrm{~Hz}, 1 \mathrm{H}), 2.84-2.73(\mathrm{~m}, 1 \mathrm{H}), 2.70-2.57(\mathrm{~m}, 1 \mathrm{H}), 2.47-2.33(\mathrm{~m}, 1 \mathrm{H}), 2.16-2.03(\mathrm{~m}, 1 \mathrm{H}), 1.92-1.76(\mathrm{~m}$, $2 \mathrm{H}), 1.25(\mathrm{t}, J=7.2 \mathrm{~Hz}, 3 \mathrm{H}) ;{ }^{13} \mathrm{C}$ NMR $\left(75 \mathrm{MHz}, \mathrm{CDCl}_{3}\right.$ )_ 206.2, 173.4, 139.7, 136.8, 132.1, 128.8, 128.7, 127.8, 61.4, 49.0, 41.0, 28.5, 20.4, 14.2; IR (film) 2939, 1730, 1681, 1254, $1188 \mathrm{~cm}^{-1}$; HRMS (EI ${ }^{+}$) calc'd for $\left[\mathrm{C}_{14} \mathrm{H}_{16} \mathrm{O}_{3}\right]^{+}: m / z 232.1100$, found 232.1095 .

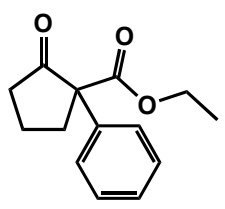

SM3. Purification by flash chromatography (25:1 hexanes/EtOAc eluent) provided the $\alpha$-arylated product (30.3 mg, $33 \%$ yield) as a clear oil: $\mathrm{R}_{\mathrm{F}} 0.42$ (3:1 hexanes/EtOAc); ${ }^{1} \mathrm{H}$ NMR $\left(300 \mathrm{MHz}, \mathrm{CDCl}_{3}\right) \delta$ 7.42-7.28 (m, 5H), 4.18 (q, $J=7.1 \mathrm{~Hz}, 2 \mathrm{H}), 2.94-2.82(\mathrm{~m}, 1 \mathrm{H}), 2.59-2.44(\mathrm{~m}, 2 \mathrm{H}), 2.42-2.27(\mathrm{~m}, 1 \mathrm{H}), 2.10-1.86(\mathrm{~m}, 2 \mathrm{H}), 1.21(\mathrm{t}, J$ $=7.0 \mathrm{~Hz}, 3 \mathrm{H}) ;{ }^{13} \mathrm{C} \mathrm{NMR}\left(75 \mathrm{MHz}, \mathrm{CDCl}_{3}\right) \delta$ 212.2, 170.9, 136.4, 128.7, 127.8, 127.6, 65.2, 62.1, 38.0, 35.1, 19.5, 14.2; IR (film) 2976, 1747, 1712, 1445, $1212 \mathrm{~cm}^{-1}$. 


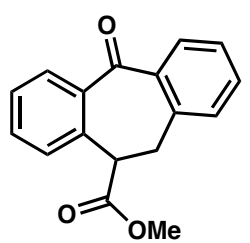

13b. Purification by flash chromatography (25:1 hexanes/EtOAc eluent) provided the desired acyl-alkylation product (63.9 mg, 61\% yield) as a clear oil: $\mathrm{R}_{\mathrm{F}} 0.31$ (3:1 hexanes/EtOAc); ${ }^{1} \mathrm{H}$ NMR (300 MHz, $\left.\mathrm{CDCl}_{3}\right)$ _ 8.10 $(\mathrm{dd}, J=7.7,1.6 \mathrm{~Hz}, 1 \mathrm{H}), 8.01(\mathrm{dd}, J=7.8,1.5 \mathrm{~Hz}, 1 \mathrm{H}), 7.53-7.30(\mathrm{~m}, 4 \mathrm{H}), 7.24-7.18(\mathrm{~m}, 2 \mathrm{H}), 4.26(\mathrm{t}, J=4.8$ $\mathrm{Hz}, 1 \mathrm{H}), 3.56(\mathrm{~d}, J=5.1 \mathrm{~Hz}, 2 \mathrm{H}), 3.50(\mathrm{~s}, 3 \mathrm{H}) ;{ }^{13} \mathrm{C} \mathrm{NMR}\left(75 \mathrm{MHz}, \mathrm{CDCl}_{3}\right)_{\text {- }}$ 194.4, 172.5, 138.8, 138.6, $138.4,137.5,132.7,131.5,130.6,130.3,129.6,128.9$, 128.0, 127.4, 52.4, 50.6, 37.8; IR (film) 2951, 1737, $1649,1599,1292,1240,1170 \mathrm{~cm}^{-1}$; HRMS $\left(\mathrm{EI}^{+}\right)$calc'd for $\left[\mathrm{C}_{17} \mathrm{H}_{14} \mathrm{O}_{3}\right]^{+}: \mathrm{m} / z$ 266.0943, found 266.0941.

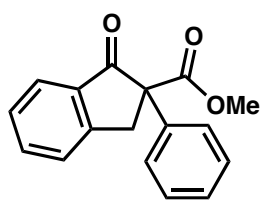

SM4. Purification by flash chromatography (25:1 hexanes/EtOAc eluent) provided the $\alpha$-arylated product (42 mg, 39\% yield) as a clear oil: $\mathrm{R}_{\mathrm{F}} 0.35$ (3:1 hexanes/EtOAc); ${ }^{1} \mathrm{H}$ NMR $\left(300 \mathrm{MHz}, \mathrm{CDCl}_{3}\right)$ _ $7.74(\mathrm{~d}, J=7.4 \mathrm{~Hz}$, 1H), 7.54 (app. dt, $J=7.4,1.2 \mathrm{~Hz}, 1 \mathrm{H}), 7.42-7.14$ (m, 7H), 4.13 (d, $J=17.3 \mathrm{~Hz}, 1 \mathrm{H}), 3.6$ (s, $3 \mathrm{H}), 3.47$ (d, $J=$ $17.3 \mathrm{~Hz}, 1 \mathrm{H}) ;{ }^{13} \mathrm{C}$ NMR $\left(75 \mathrm{MHz}, \mathrm{CDCl}_{3}\right)_{\text {_ }}$ 200.4, 171.2, 138.9, 135.9, 135.2, 128.9, 128.5, 128.2, 127.8, 127.5, 126.4, 125.3, 65.6, 53.5, 41.0; IR (film) 2952, 1745, 1716, 1606, $1211 \mathrm{~cm}^{-1}$; HRMS (EI ${ }^{+}$) calc'd for $\left[\mathrm{C}_{17} \mathrm{H}_{14} \mathrm{O}_{3}\right]^{+}: \mathrm{m} / z$ 266.0943, found 266.0934 .

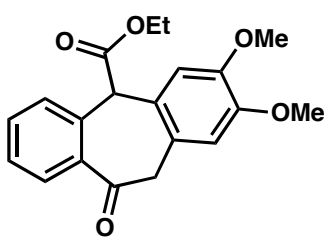

13c. Purification by flash chromatography (1:1 hexanes/EtOAc eluent) provided the desired acyl-alkylation product (75.7 mg, 65\% yield) as a clear oil: $\mathrm{R}_{\mathrm{F}} 0.12$ (3:1 hexanes/EtOAc); ${ }^{1} \mathrm{H}$ NMR $\left(300 \mathrm{MHz}, \mathrm{CDCl}_{3}\right){ }_{-} 8.10$ $(\mathrm{dd}, J=7.7,1.6 \mathrm{~Hz}, 1 \mathrm{H}), 7.52-7.30(\mathrm{~m}, 3 \mathrm{H}), 6.87(\mathrm{~s}, 1 \mathrm{H}), 6.76(\mathrm{~s}, 1 \mathrm{H}), 4.88(\mathrm{~s}, 1 \mathrm{H}), 4.43(\mathrm{~d}, J=14.8 \mathrm{~Hz}, 1 \mathrm{H})$, 4.24-4.13 (m, 2H), 3.88 (s, 3H), 3.85 (s, 3H), 3.72 (d, J=15.4 Hz, 1H), 1.19 (t, J=7.2 Hz, $3 \mathrm{H})$; ${ }^{13} \mathrm{C}$ NMR $(75$ $\left.\mathrm{MHz}, \mathrm{CDCl}_{3}\right)_{-} 194.8,171.4,148.8,148.0,139.9,134.8,133.1,131.3,131.0,129.8,128.3,124.7,114.0,113.8$, 62.1, 59.4, 56.3, 56.2, 49.5, 14.4; IR (film) 2978, 2937, 1728, 1673, 1598, 1518, 1262, 1230, 1201, 1112,1025 $\mathrm{cm}^{-1}$; HRMS (EI $)$ calc'd for $\left[\mathrm{C}_{20} \mathrm{H}_{20} \mathrm{O}_{5}\right]^{+}: \mathrm{m} / z$ 340.1311, found 340.1326. 


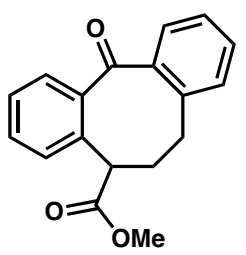

13d. Purification by flash chromatography (15:1 hexanes/EtOAc eluent) provided the desired acyl-alkylation product (50 mg, 45\% yield) as a clear oil: $\mathrm{R}_{\mathrm{F}} 0.39$ (3:1 hexanes/EtOAc); ${ }^{1} \mathrm{H}$ NMR $\left(300 \mathrm{MHz}, \mathrm{CDCl}_{3}\right){ }_{-} 8.19$ $(\mathrm{dd}, J=7.8,1.5 \mathrm{~Hz}, 1 \mathrm{H}), 7.94(\mathrm{dd}, J=7.7,1.6 \mathrm{~Hz}, 1 \mathrm{H}), 7.57-7.49$ (m, 2H), 7.47-7.30 (m, 2H), 7.24-7.16 (m, 2H), $3.88(\mathrm{dd}, J=11.6,4.9 \mathrm{~Hz}, 1 \mathrm{H}), 3.62(\mathrm{~s}, 3 \mathrm{H}), 2.71-2.53(\mathrm{~m}, 2 \mathrm{H}), 2.20-1.95(\mathrm{~m}, 2 \mathrm{H}) ;{ }^{13} \mathrm{C} \mathrm{NMR}(125 \mathrm{MHz}$, $\left.\mathrm{CDCl}_{3}\right)_{-} 194.3,173.7,141.3,140.7,139.2,136.7,133.9,133.0,131.3,131.1,130.8,127.7,127.2,126.3,52.3$, 45.3, 35.6, 30.6; IR (film) 2951, 1736, 1638, 1595, 1292, 1254, $1219 \mathrm{~cm}^{-1}$; HRMS (EI ${ }^{+}$) calc'd for $\left[\mathrm{C}_{18} \mathrm{H}_{16} \mathrm{O}_{3}\right]^{+}$: $m / z 280.1100$, found 280.1108 .<smiles>COC(=O)C1(c2ccccc2)CCc2ccccc2C1=O</smiles>

SM5. Purification by flash chromatography (15:1 hexanes/EtOAc eluent) provided the $\alpha$-arylated product (61.5 mg, 55\% yield) as a clear oil: $\mathrm{R}_{\mathrm{F}} 0.31$ (3:1 hexanes/EtOAc); ${ }^{1} \mathrm{H}$ NMR $\left(300 \mathrm{MHz}, \mathrm{CDCl}_{3}\right)_{-} 8.15(\mathrm{dd}, J=8.0,1.3$ $\mathrm{Hz}, 1 \mathrm{H}), 7.50-7.42(\mathrm{~m}, 1 \mathrm{H}), 7.38-7.28(\mathrm{~m}, 4 \mathrm{H}), 7.25-7.14(\mathrm{~m}, 3 \mathrm{H}), 3.75(\mathrm{~s}, 3 \mathrm{H}), 3.08-2.91(\mathrm{~m}, 2 \mathrm{H}), 2.88-2.70$ $(\mathrm{m}, 2 \mathrm{H}) ;{ }^{13} \mathrm{C} \mathrm{NMR}\left(125 \mathrm{MHz}, \mathrm{CDCl}_{3}\right) \ldots$ 194.7, 172.3, 143.2, 136.4, 133.9, 132.3, 128.9, 128.7, 128.5, 127.9, 127.9, 127.1, 63.7, 53.0, 32.6, 26.0; IR (film) 2951, 1732, 1682, 1600, $1253 \mathrm{~cm}^{-1}$; HRMS (EI ${ }^{+}$) calc'd for $\left[\mathrm{C}_{18} \mathrm{H}_{16} \mathrm{O}_{3}\right]^{+}: m / z 280.1100$, found 280.1107 .

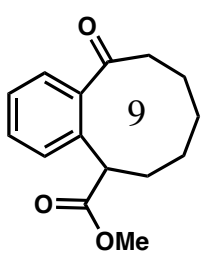

13e. Purification by flash chromatography (20:1 hexanes/EtOAc eluent) provided the desired acyl-alkylation product (67.6 mg, 69\% yield) as a clear oil: $\mathrm{R}_{\mathrm{F}} 0.29$ (3:1 hexanes/EtOAc); ${ }^{1} \mathrm{H}$ NMR $\left(300 \mathrm{MHz}, \mathrm{CDCl}_{3}\right){ }_{-} 7.40-$ $7.17(\mathrm{~m}, 4 \mathrm{H}), 3.95(\mathrm{dd}, J=11.7,4.8 \mathrm{~Hz}, 1 \mathrm{H}), 3.66(\mathrm{~s}, 3 \mathrm{H}), 2.96-2.85(\mathrm{~m}, 1 \mathrm{H}), 2.84-2.72(\mathrm{~m}, 1 \mathrm{H}), 2.07-1.80(\mathrm{~m}$, 4H), 1.77-1.45 (m, 4H); ${ }^{13} \mathrm{C}$ NMR (75 MHz, $\left.\mathrm{CDCl}_{3}\right)_{-}$211.4, 174.5, 143.3, 134.9, 130.1, 127.9, 127.2, 124.9, 52.3, 46.0, 43.5, 32.3, 26.0, 25.4, 23.8; IR (film) 2936, 2860, 1732, 1693, 1435, 1249, $1201 \mathrm{~cm}^{-1}$; $\mathrm{HRMS}_{(\mathrm{EI}}^{+}$) calc'd for $\left[\mathrm{C}_{15} \mathrm{H}_{18} \mathrm{O}_{3}\right]^{+}: \mathrm{m} / z$ 246.1256, found 246.1255 . 


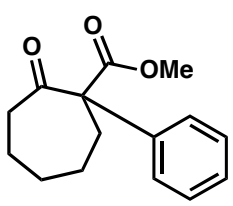

SM6. Purification by flash chromatography (15:1 hexanes/EtOAc eluent) provided the $\alpha$-arylated product (29.9 mg, $30 \%$ yield) as a clear oil: $\mathrm{R}_{\mathrm{F}} 0.39$ (3:1 hexanes/EtOAc); ${ }^{1} \mathrm{H}$ NMR $\left(300 \mathrm{MHz}, \mathrm{CDCl}_{3}\right) \delta$ 7.37-7.28 (m, 3H), 7.22-7.18 (m, 2H), 3.69 (s, 3H), 2.83-2.72 (m, 2H), 2.61-2.54 (m, 2H), 2.24-2.13 (m, 2H), 2.00-1.56 (m, 4H); ${ }^{13} \mathrm{C}$ NMR $\left(125 \mathrm{MHz}, \mathrm{CDCl}_{3}\right) \delta$ 208.89, 172.8, 139.1, 128.7, 127.8, 127.7, 68.5, 52.8, 42.2, 34.0, 30.7, 26.7, 25.8; IR (film) 2928.79, 1729, 1703, 1490, 1446, 1228, $1155 \mathrm{~cm}^{-1}$.

\section{Independent Chemical Correlation / Structural Proof}

Acyl-alkylation product $9 \mathbf{a}$ was independently prepared according to a literature procedure. ${ }^{14}$ The product obtained through our methodology was identical by all spectroscopic data to the compound prepared by this alternative method. Spectroscopic data for acyl-alkylation product $\mathbf{9 f}$ was identical to all the reported data in the literature. ${ }^{15}$ 


\section{References}

${ }^{1}$ Pena, D.; Perez, D.; Guitian, E.; Castedo, L. J. Am. Chem. Soc. 1999, 121, 5827-5828.

2 Yoshikawa, E.; Radhakrishnan, K. V.; Yamamoto, Y. J. Am. Chem. Soc. 2000, 122, 7280-7286.

3 Witiak, D. T.; Kim, S. K.; Tehim, A. K.; Sternitzke, K. D.; McCreery, R. L. et al. J. Med. Chem. 1988, 31, 1437-1445.

4 Jung, M. E.; Hagenah, J. A. J. Org. Chem. 1987, 52, 1889-1902.

5 Phukan, P.; Mohan, J. Madan; and Sudalai, A. J. Chem. Soc., Perkin Trans. 1 1999, 24, 3685-3690.

${ }^{6}$ Selwood, D. L.; Brummell, D. G.; Budworth, J. ; Burtin, G. E.; Campbel, R. O.; Chana, S. S.; Charles, I. G.; Fernandez, P. A.; Glen, R. C.; Goggin, M. C.; Hobbs, A.J.; et al. J. Med. Chem. 2001, 44, 78-93.

7 Zheng, S.-L.; Yu, W.-Y.; Che, C.-M. Org. Lett. 2002, 4, 889-892.

8 Taber, D. F.; Deker, P. B.; Gaul, M. D. J. Am. Chem. Soc. 1987, 109, 7488-7494.

${ }^{9}$ Doyle, M. P.; Westrum, L. J.; Wolthuis, W. N. E.; See, M. M.; Boone, W. P.; Bagheri, V.; Pearson, M. M. J. Am. Chem. Soc. 1993, 115, 958-964.

10 Nudelman, A.; Kelner, R.; Broida, N.; Gottlieb, H. E. Synthesis 1989, 5, 387-388.

11 Emelen, K. V.; Wit, T. D.; Hoornaert, G. J.; Compernolle, F. Org. Lett. 2000, 2, 3083-3086.

12 Taylor, J. B. et al. J. Med. Chem. 1970, 13, 1226-1227.

13 Brown, D. S.; Marples, B. A.; Smith, P.; Walton, L. Tetrahedron 1995, 51, 3587-3606.

14 Cruces, J.; Estevez, J. C.; Castedo, L.; Estevez, R. J. Tetrahedron Lett. 2001, 42, 4825-4828.

15 Rupard, J. H.; Paulis, T.; Janowsky, A.; Smith, H. E. J Med. Chem. 1989, 32, 2261-2268. 